ARTICLE

\title{
The effect of cultural transmission on shared sign language persistence
}

\author{
Katie Mudd ${ }^{1 凶}$, Connie de $\operatorname{Vos}_{(10}{ }^{2} \&$ Bart de Boer ${ }^{1}$
}

\begin{abstract}
In this paper, we revisit a mathematical model of sign language persistence by Aoki and Feldman (Theor Popul Biol 39(3):358-372, 1991), which investigates the evolution of genes causing deafness, affected by an assortative mating parameter, and the cultural transmission of sign language. To assess their model, we reimplement it as an agent-based simulation to be able to easily represent structured relationships in a finite population. We study the persistence of shared sign languages, a categorization of sign languages, which are typically shared by deaf and hearing members of a small community with a high incidence of hereditary deafness (Nyst, 2012. Shared sign languages. Sign language: An international handbook, pp. 552-574). We observe how shared sign language persistence is affected by hearing signers, marriage patterns, and various modes of sign language transmission: vertical, horizontal, oblique, and grandparental transmission. In contrast to Aoki and Feldman's (Theor Popul Biol 9(3):358-372, 1991) finding that modes of transmission other than vertical are negligible, in the agent-based model we find that adding modes of transmission helps to ensure shared sign language persistence. A better understanding of sign language persistence has relevance for processes of cultural evolution, (sign language) linguistics, and language endangerment.
\end{abstract}

\footnotetext{
${ }^{1}$ Vrije Universiteit Brussel, Brussel, Belgium. ${ }^{2}$ Tilburg University, Tilburg, The Netherlands. ${ }^{凶}$ email: katie.mudd@ai.vub.ac.be
} 


\section{Introduction}

uman evolution has been shaped by interactions between genes and culture (Aoki and Feldman, 1991). The theory of gene-culture coevolution posits that genes and culture are interacting systems, in which offspring inherit both genetic and cultural material (Feldman and Cavalli-Sforza, 1976). Models of gene-culture coevolution typically find faster rates of change than models from conventional population genetics (Feldman and Laland, 1996). Additionally, in response to human cultural activity, genes (sometimes deleterious from a purely biological perspective) have undergone positive selection. It has been estimated that up to $10 \%$ of the human genome may be influenced by positive selection (Williamson et al., 2007), a few examples being lactase persistence (Holden and Mace, 2009; Bersaglieri et al., 2004), handedness (Laland et al., 1995) and the evolution of language-facilitating genes like FOXP2 (Coop et al., 2008). However, it is difficult to thoroughly examine many of these cases; the phenotype and cultural conditions favoring a given phenotype are no longer available for examination, as these changes have taken place at various points in the course of human evolution.

In this article, we revisit a unique case of gene-culture coevolution for which we have recent to present-day genetic and cultural evidence, namely, the coevolution of sign language and hereditary deafness. Some sign languages in use today have persisted for over 200 years (e.g. ASL: Woll et al., 2001), while others are very young, such as Nicaraguan Sign Language which is not more than 40 years old (Senghas, 2005). Certain sign languages have been documented in great detail by geneticists, anthropologists, sociologists, and linguists, providing ample material to model the factors contributing to sign language persistence (for examples, see sociolinguistic sketches in Zeshan and de Vos, 2012; for further discussion see Mudd et al., in press). In addition, models of gene-culture coevolution have typically been undertaken without knowledge of the underlying genes involved (Laland et al., 2010). But, the genes involved in hereditary deafness are fairly well understood (Petit, 1996); hence it is possible to precisely model the genetic and cultural factors involved in hereditary deafness and sign language use.

Aoki and Feldman (1991, 1994) and Feldman and Aoki (1992) investigated this unique case, where sign language persistence (i.e. protection from loss) is said to depend on genetic factors and cultural factors, such as marriage and sign language transmission. In the present research, we re-evaluate these models to further investigate what conditions favor the persistence of shared sign languages (i.e. sign languages that have emerged in enclaves with a high incidence of hereditary deafness and are typically shared by both deaf and hearing members of a community, Nyst, 2012). In order to re-evaluate their work, we conducted a parameter exploration using an agent-based model. With an increasing amount of sign language documentation, it is possible to make more accurate and data-driven models of sign language persistence. Here, we present a first step taken to make such a model. We focus our study mainly on the effect of the various modes of cultural transmission of sign language, but also on the role of hearing individuals and marriage patterns on sign language persistence.

\section{Modes of cultural transmission}

Cavalli-Sforza and Feldman (1981) introduce and outline various modes of cultural transmission: vertical transmission is from parents to offspring, oblique transmission is from an individual of the parental generation (but not the parents) to the offspring, and horizontal transmission is between individuals of the same generation. These modes of cultural transmission have been at the heart of cultural evolution research. The general finding is that different modes of cultural transmission have different effects on a given cultural entity; for example, Acerbi and Parisi (2006) consider the effect of horizontal and oblique cultural transmission on the cultural emergence of effective behaviors. They posit that horizontal transmission introduces random noise (and thus, adds more variability) in the process of cultural transmission, whereas inter-generational transmission (here, vertical and oblique transmission) is more faithful. Gong et al. (2010) and Gong and Shuai (2016) confirm this general finding in a study of the convergence of linguistic knowledge, finding that vertical, oblique, and grandparental transmission help to preserve the original language while horizontal transmission introduces changes to it.

The study of language evolution has focussed specifically on the contributions of the different modes of cultural transmission on linguistic structure, using a prominent methodology in the study of language evolution called the iterated learning model. In this paradigm, individuals acquire a behavior from another individual who has acquired that behavior in the same way. As learners do not have access to the entire linguistic repertoire of their tutor, they are forced to generalize (Kirby and Hurford, 2002). Using the iterated learning model, the emergence of compositionality can be explained by cultural transmission alone, and does not require a biological explanation (Kirby, 2001). This paradigm initially implemented vertical transmission between single-agent generations (e.g. Kirby and Hurford, 2002), and was expanded upon to consider larger generations (e.g. Smith and Hurford, 2003), horizontal transmission (e.g. Kirby, 2000) and both vertical and horizontal transmission (Theisen-White et al., 2011). Though these experiments were initially conducted using mathematical and computational modeling, more recently these models have been tested experimentally in the lab with participants learning and producing nonce words (Kirby et al., 2008) and using the silent-gesture paradigm (i.e. improvised sign language, Motamedi et al., 2019). The main conclusions from this line of research are that horizontal transmission makes language efficient, while inter-generational (vertical and oblique) transmission makes language systematic. Emerging sign languages provide naturalistic evidence of the role of these transmission types: for example, horizontal transmission in one single generation can lead to sign language emergence (Senghas et al., 2004) and subsequent learners exhibit various degrees of linguistic cohesiveness depending on which types of transmission (vertical, horizontal or both) they are exposed to (Gagne, 2017).

Models, laboratory experiments, and naturalistic data have begun to clarify the role of the different modes of cultural transmission on linguistic features. However, still unclear, is the role of these various transmission modes on language persistence. In this paper, we focus on the role of vertical, horizontal, oblique, and grandparental transmission on sign language persistence. Depending on the society, different cultural elements may be acquired by varying proportions of the outlined modes of cultural transmission (for an example, see Hewlett and Cavalli-Sforza, 1986). A better understanding of the role of different modes of cultural transmission on the persistence of a cultural trait has implications for the study of language vitality and endangerment, cultural evolution and (sign language) linguistics.

\section{Models of sign language persistence}

A number of models have addressed sign language persistence (Aoki and Feldman, 1991, 1994; Feldman and Aoki, 1992; Arnos et al., 2008; Nance et al., 2000; Nance and Kearsey, 2004). The focus of these models has largely been on assortative mating based on deaf/hearing status (i.e. to what extent deaf individuals 
have a tendency to have offspring with deaf individuals, and to what extent hearing individuals have a tendency to have offspring with hearing individuals). I will briefly outline some of the components and contributions from two key models.

Aoki and Feldman (1991) consider how genetic and cultural sign language transmission affect the persistence of a sign language. Their model assumes that deaf individuals are more likely to acquire a sign language than hearing individuals, and that (unlike hereditary deafness) sign language cannot skip a generation and reappear by vertical transmission alone. The main findings are the following: sign language persistence is more easily achieved with (a) a high proportion of hearing individuals acquiring the sign language, (b) a high proportion of recessive alleles for deafness in the population, and (c) a high value of assortative mating for deaf/hearing status. To clarify, a high value of assortative mating for deaf/hearing status results in predominantly deaf-deaf mating and hearing-hearing mating (in other words, like has offspring with like). A low value of assortative mating means indifference towards the deaf/hearing status of one's partner. Additionally, Aoki and Feldman (1991) test the effect of other factors on sign language persistence, such as the role of oblique and horizontal cultural transmission of the sign language, and conclude that their effects are negligible on sign language persistence.

Aoki and Feldman (1991) focus one one specific case, with vertical transmission alone from deaf parents to deaf offspring, in a population with a single monogenic form of recessive deafness. In this case, the conditions for signers to persist in a population is

$$
2 l_{\mathrm{c}}[(1-m) q+m]>1
$$

where $l_{c}$ is the probability of a deaf child acquiring a sign language with only one signing parent, $m$ is the proportion of assortative mating for deaf/hearing status, and $q$ is the frequency of recessive alleles causing deafness. According to this model, the inequality (1) cannot be satisfied if $m$ is small (Aoki and Feldman, 1991). In other words, if mating is random with regards to deaf/hearing status, the model does not predict sign language persistence.

To expand on their previous model, Feldman and Aoki (1992) describe a method to account for the cultural transmission of a sign language from grandparents to grandchildren. Their method consists of two overlapping mother-father-child triplets, allowing sign language transmission between the maternal grandmother and grandchild. For this model, the following assumptions are (a) a high rate of assortative mating, (b) the absence of horizontal or oblique transmission, and (c) sign language transmission only between deaf individuals. Given this set of assumptions, Feldman and Aoki (1992) find that grandmaternal transmission bolsters sign language persistence in cases where vertical transmission alone was insufficient.

It is important to note that these models were developed with Western populations in mind, evidenced by cited literature and modeling decisions. To begin, Aoki and Feldman (1991) cite the high proportion of deaf-deaf marriages in the US and in England (Kyle and Woll, 1985) and the incidence of deafness in the British Isles, representing $0.1 \%$ of the population (Fraser, 1976). Next, horizontal and oblique transmission are modeled as transmission between random individuals in an individual's same generation and parental generation, respectively, as opposed to modeling transmission within the family (i.e. horizontal transmission between siblings, and oblique transmission between an individual and their aunts/uncles). Horizontal and oblique transmissions are modeled this way presumably to represent sign language transmission occurring in deaf schools (Feldman and Aoki, 1992).

In sum, Aoki and Feldman (1991) develop a mathematical model of sign language persistence suited for Western populations. No values from existing sign language communities are applied to the model by Aoki and Feldman (1991). With increased documentation of sign language communities over the past decades, we can now reconsider this model with inputs from real sign language communities.

Gialluisi et al. (2013) apply values from the Al-Sayyid Bedouin sign language (ABSL) community to Aoki and Feldman's model (1). ABSL emerged over 80 years ago in a Bedouin community in the Negev region of Israel. In this community of approximately 3500 individuals, $\sim 2 \%$ are congenitally deaf. As deafness in this community is a hereditary trait, deaf individuals are typically born to an extended family with deaf members and signers. Additionally, hearing individuals are prominent members of the signing community. Deaf individuals are not stigmatized and they always marry a hearing individual (Senghas, 2005; Kisch, 2012).

Taking into account the characteristics of the community in which ABSL emerged, Gialluisi et al. (2013) set the inputs to Aoki and Feldman's (1991) model (1). For vertical sign language transmission from parents to deaf offspring, as all deaf individuals learn to sign in the community, $l_{c}$ is set to 1 . As a conservative estimate of the frequency of the recessive deafness allele, $q$ is set to 0.18 . Lastly, because in this community it is the norm for deaf individuals to marry a hearing individual (Kisch, 2012), $m$ is set to 0 . When these figures are applied to the mathematical model, the sign language is incorrectly predicted not to persist. Hence, other factors besides assortative mating and vertical sign language transmission to deaf individuals must be taken into account in order to explain sign language persistence in this community.

Gialluisi et al. (2013) highlight that previous models of sign language persistence have emphasized the role of assortative mating for deafness, and neglected the role of other features present in non-Western signing communities (e.g. endogamy, consanguineous marriages and the cultural integration of deaf individuals). Thus, these models neglect a group of signing communities typically called shared sign languages (Nyst, 2012). These sign languages typically emerge in insular communities with a high incidence of hereditary deafness (Nyst, 2012; Meir et al., 2010). In some of these communities, marriages between deaf individuals are uncommon or not allowed, as in the Adamarobe sign language community (AdaSL) (Kusters, 2012) and in the ABSL community (Kisch, 2012). It is unlikely that marriage patterns are the key to sign language persistence in these communities (Gialluisi et al., 2013), as is emphasized in previous models.

Because of the high proportion of hearing signers typically taking part in the signing community in shared sign languages (Nyst, 2012), this may be one of the missing links in explaining the persistence of these languages. Because Gialluisi et al. (2013) do not have data about the likelihood of hearing individuals acquiring ABSL, they do not consider the role of hearing signers in the persistence of ABSL. In addition, the model they used only considered vertical transmission. In these communities, it is unrealistic that vertical transmission alone allows for sign language persistence-more modes of cultural transmission should be considered. There is undoubtedly a plethora of other relevant aspects of these communities that should be taken into account to explain sign language persistence in shared signing communities, as highlighted by Gialluisi et al. (2013). As a detailed example, Lutzenberger (submitted) outlines the recent developments in the Kata Kolok community likely affecting the persistence of the sign language, such as the negative influences of the media and the decreasing number of deaf children being born, and the positive influences of education and sign language documentation. Though we acknowledge these factors are undoubtedly important, for the sake of having a simple, interpretable model (see Smaldino, 2017), we focus on modeling different modes of sign language transmission, additionally considering the role of 
hearing signers and assortative marriage for deaf/hearing status. In this exploration, we take into account features of shared signing communities and apply typical values from them to the agent-based model created.

The focus of previous models of sign language persistence has been on the role of assortative mating for deaf/hearing status. What is yet unexplored is the role of various structured transmission types on sign language persistence-vertical, horizontal, oblique, and grandparental transmission. Additionally, in the case of shared sign languages, the role of hearing individuals as participants in the linguistic community cannot be ignored in models of sign language persistence. In shared sign language communities, deaf individuals always acquire the sign language if possible, and many hearing individuals do as well. Here, we consider the effect of hearing individuals as participants in the signing community. In previous models of sign language persistence, their role was not sufficiently considered.

Though Aoki and Feldman (1991) and Feldman and Aoki (1992) do explore the role of various transmission types, they make several limiting assumptions. First, they assume that only a small proportion of hearing individuals will acquire the sign language. Second, likely due to the limitations of mathematical modeling, grandparental transmission considers only the maternal grandmother. And third, horizontal and oblique transmission are not structured; in other words, they occur between random individuals. What is more realistic in shared signing communities is that language transmission occurs in a structured manner within the family. Hence, here we model horizontal transmission as occurring between siblings and oblique transmission as occurring between an individual and their aunts and uncles.

The present paper makes the following contributions, largely afforded by reimplementing Aoki and Feldman's (1991) mathematical model as an agent-based model. Here, we

a. consider realistic values from shared sign languages as input parameters, such as the population size and prevalence of deaf alleles

b. model horizontal and oblique transmission as structured (as opposed to random transmission)

c. allow for sign language transmission from all grandparents

The paper is structured as follows; the next section provides details of the agent-based model. Subsequently, we present results from two experiments with realistic values of features from shared signing communities. In experiment 1, with vertical transmission alone, we explore the effect of marriage patterns on sign language persistence. In experiment 2 , we consider the role of various methods of cultural transmission within the familyvertical, horizontal, oblique and grandparental transmission-on sign language persistence, as well as the role of hearing signers. For experiment 2 we focus on the case highlighted by Gialluisi et al. (2013) for ABSL, namely where marriages between deaf individuals do not occur. After experiment results and discussion, we proceed with a general discussion.

\section{Model}

This agent-based model can be used to investigate how sign language persistence is affected by various factors, including (but not limited to) marriage patterns, population size, proportion of deafness, and modes of sign language transmission. In the current investigation, we focus on various modes of sign language transmission, and also on the effect of marriage patterns and hearing signers on sign language persistence. One version of this model is originally described in Mudd et al. (in press), where the focus was on moving towards a realistic model of shared signing communities; to do so, the entire family unit was considered for

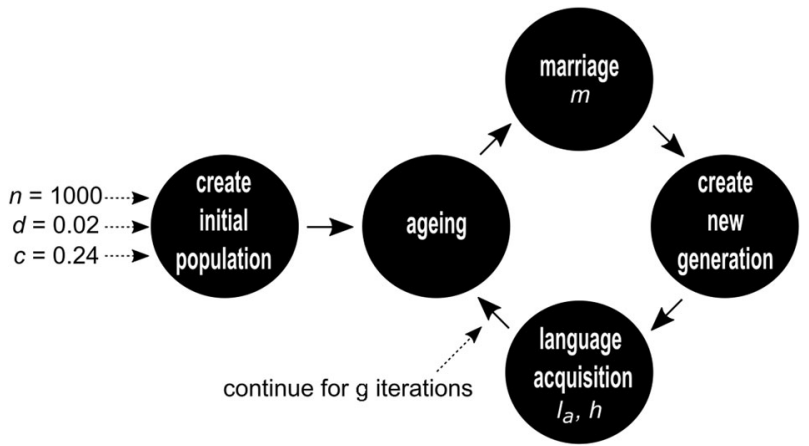

Fig. 1 Visualization of the agent-based model of sign language

persistence. To create the initial population, the input parameters needed are: the size of a generation $(n)$, the number of deaf agents $(d)$ and the number of hearing agents carrying a deaf allele (c). Next, continuing for $g$ iterations, agents age (and are removed from the population after three years), agents marry based on the assortative marriage parameter $(m)$, a new generation is created from the offspring of couples, and language acquisition occurs based on the mode of language transmission $\left(I_{a}\right)$ and the proportion of hearing signers who acquire the sign language if possible $(h)$.

language transmission, and the effect of various other factors (such as population size) on sign language persistence were considered. In contrast, the focus of the current modeling effort is to explicitly test certain modeling decisions made in the models by Aoki and Feldman (1991) and Feldman and Aoki (1992), such as the proportion of hearing signers who acquire the sign language. The description of the model given here is based on the one given in Mudd et al. (in press).

The model description follows the ODD (Overview, Design concepts, Details) protocol for describing agent-based simulations (Grimm et al., 2006, 2010). The model code is available at https:// doi.org/10.6084/m9.figshare.11522589.v5.

Purpose. The purpose of this model is to explore the factors allowing for shared sign languages to persist. As shown in Fig. 1, the agent-based model takes the following values as input parameters: the number of deaf agents $(d)$, the number of hearing agents carrying a deaf allele $(c)$, the size of a generation $(n)$, an assortative marriage value $(m)$, the mode of language transmission $\left(l_{\mathrm{a}}\right)$ and the proportion of hearing signers who acquire the sign language if possible $(h)$ (implemented in the language acquisition part of the model). Deaf alleles ( $d$ and $c$ ) determine the persistence and transmission of deafness while sign language transmission is determined by the method of sign language transmission used $\left(l_{\mathrm{a}}\right)$ and the number of hearing signers who acquire the sign language if possible $(h)$. The assortative marriage parameter $(m)$ presents a link, affecting both sign language transmission and the transmission of recessive alleles for deafness.

Entities, state variables, and scales. The only entity in the model is the agent. Agents consist of the following: identity number, age, sex, genes, parents, partner and sign ability. Agents live for three generations $(0,1,2)$, and at age 1 most agents get married and reproduce. At any age, agents can transmit or acquire the sign language.

Process overview and scheduling. The set-up of the model is outlined in initialization below. After the initialization phase, each time step consists of the processes outlined in Table 1. For details of these processes, see "Submodel" section. A schematic overview of the order of processes and parameter input is 


\begin{tabular}{|c|c|c|}
\hline Process & Pseudo-code & Parameters \\
\hline $\begin{array}{l}\text { Create initial } \\
\text { generation }\end{array}$ & $\begin{array}{l}\text { create } n \text { agents } \\
\text { initialize according to gene } \\
\text { inheritance } \\
\text { if agent is deaf } \\
\text { it acquires the sign language } \\
\text { for each agent } \\
\text { increase age by } 1 \\
\text { if age is } 3 \\
\text { remove agent from population }\end{array}$ & $n, d, c$ \\
\hline Marriage & $\begin{array}{l}\text { select agents with age } 1 \\
\text { perform assortative marriage with } \\
\text { value } m\end{array}$ & $m$ \\
\hline $\begin{array}{l}\text { Create new } \\
\text { generation }\end{array}$ & $\begin{array}{l}\text { repeat } n \text { times } \\
\text { randomly choose couple } \\
\text { create agent with selected couple } \\
\text { as parents }\end{array}$ & $n$ \\
\hline Gene inheritance & $\begin{array}{l}\text { for each new agent } \\
\text { randomly acquire one allele from } \\
\text { each parent } \\
\text { if the agent acquires two deaf } \\
\text { alleles } \\
\text { it is deaf } \\
\text { else } \\
\text { it is hearing }\end{array}$ & \\
\hline Language acquisition & $\begin{array}{l}\text { for each agent } \\
\text { given } I_{a} \text {, perform language } \\
\text { acquisition } \\
\text { if deaf } \\
\text { acquire the sign language } \\
\text { if hearing } \\
\text { acquire the sign language with } \\
\text { probability } h\end{array}$ & $l_{a}, h$ \\
\hline
\end{tabular}

provided in Fig. 1. The number of agents in one generation is referred to as $n$. The full population $n_{\text {total }}$ is comprised of three generations (aged $0,1,2)$ alive at once.

Initialization. The model takes the following input parameters: the number of deaf agents $(d)$, the number of hearing agents carrying a deaf allele $(c)$, the size of one generation $(n)$, the value for assortative marriage $(m)$, the mode of language transmission $\left(l_{\mathrm{a}}\right)$, the proportion of hearing signers who acquire the sign language if possible $(h)$ and how many generations the model runs for $(g)$. The initial number of agents in the model is one generation $(n)$, equaling a third of the total population size of a sign language community, calculated by $n_{\text {total }} / 3$. The gene distribution for these agents is set according to $d$ and $c$ (see section "Submodel gene inheritance"). In the first generation, all deaf agents are signers.

Submodel gene inheritance. When an agent is born, it inherits one allele from each of its parents. From each parent, the agent inherits either an allele causing deafness (a recessive allele (d)) or an allele that does not cause deafness (a dominant allele (D)). As the model pertains to recessive hereditary deafness, only agents who acquire two alleles causing deafness, homozygous recessive (dd), have a deaf phenotype. Agents who acquire only one allele causing deafness, heterozygous $(\mathrm{dD})$ or $(\mathrm{Dd})$, are carriers of the allele causing deafness, and, along with agents acquiring no alleles causing deafness, homozygous dominant (DD), have a hearing phenotype.

\section{Table 2 Summary of experiment 2 results.}

\begin{tabular}{|c|c|}
\hline$\underline{l_{a}}$ & $h$ \\
\hline V & $>0.4$ \\
\hline $\mathrm{VH}$ & $>0.3$ \\
\hline VO & $>0.1$ \\
\hline VG & $>0.1$ \\
\hline VHO & $>0.1$ \\
\hline VHG & $>0.1$ \\
\hline VOG & $>0$ \\
\hline VHOG & $>0$ \\
\hline
\end{tabular}

For the initialization of genes in the first generation, input parameters needed are the size of a generation $(n)$, the number of deaf agents $(d)$, and the proportion of hearing agents carrying a deaf allele $(c)$. The number of deaf agents in the initial generation is calculated by $n \cdot d$, and the number of hearing carriers of the deaf allele is calculated by $n \cdot c$. The remaining agents in the population are hearing agents carrying no deaf alleles.

Submodel language acquisition. Acquiring sign language is modeled as a binary variable; thus, agents either acquire the sign language or they do not acquire the sign language. Agents cannot unlearn the sign language once it is acquired. Depending on the language acquisition method specified $\left(l_{\mathrm{a}}\right)$, an agent has the opportunity to acquire the sign language from their parents via vertical transmission $(\mathrm{V})$, their siblings via horizontal transmission $(\mathrm{H})$, their aunts and uncles via oblique transmission $(\mathrm{O})$ and their grandparents via grandparental transmission $(\mathrm{G})$. Language acquisition is modeled as combinations of these transmission types; the possible model inputs for language acquisition are the following: vertical transmission alone (V), vertical and horizontal transmission $(\mathrm{VH})$, vertical and oblique transmission (VO), vertical and grandparental transmission (VG), vertical, horizontal, and oblique transmission (VHO), vertical, horizontal, and grandparental transmission (VHG), vertical, oblique, and grandparental transmission (VOG), or vertical, horizontal, oblique and grandparental transmission (VHOG) (Table 2).

In language acquisition, deaf agents always acquire the sign language if possible (i.e. if an agent knows the sign language given the transmission method specified). Meanwhile, if possible, hearing agents acquire the sign language with probability $h$.

Submodel assortative marriage. All agents who are age 1 are eligible to get married. The assortative marriage value $(\mathrm{m})$ specifies the proportion of agents seeking a partner with the same phenotype. Hence, we calculate the number of deaf agents in a deaf-deaf marriage and hearing agents in a hearing-hearing marriage. The number of deaf agents that marry a deaf agent is calculated by the number of deaf agents in a generation $n_{\mathrm{d}}=n \cdot d$, multiplied by the assortative marriage value $(m)$, yielding $n_{\mathrm{d}} \cdot m$. The number of hearing agents that marry a hearing agent is calculated by finding the number of eligible hearing agents in a generation, $n_{h}=n-n_{\mathrm{d}}$, and multiplied by the assortative marriage value $(m)$, yielding $n_{\mathrm{h}} \cdot m$. The remaining agents in a given generation, $n-\left(n_{\mathrm{d}} \cdot m+n_{\mathrm{h}} \cdot m\right)$, are randomly married with respect to deafness. In addition to the possibility of specifying the proportion of $m$, it is also possible for $m$ to be set to no_dd, where marriages between deaf agents are prohibited. For all possible settings of $m$ (values 0 to 1 and no_dd), marriages between siblings are prohibited. It is possible that due to constraints (no sibling marriages, female-male marriages and the marriage type specified) some agents are left unmarried. 


\section{Results and discussion}

All results of model simulations presented here are of 100 model runs. The model output consists of agent characteristics recorded at each time step. In the current analysis, we focus on the proportion of signers in the population. In the figures presented in the following sections, the dark line represents the median and the shaded area represents the first and third quartiles, calculated at each time step. If the error region (i.e. the shaded area representing the first and third quartiles) of one experiment overlaps with the median (represented by the dark line) of another experiment, then there is no significant difference in result between the two.

In the following experiments, the size of one generation $(n)$ is 1000 , totaling 3000 for the entire population at once. In the first time step of the model there are 1000 agents (i.e. generation one), in the second time step of the model there are 2000 agents (i.e. generation one and two) and in the third time step of the model there are 3000 agents (generation one, two and three). From the third generation onward the population size is 3000. Agents live for three generations. In line with previous models of sign language persistence (Aoki and Feldman, 1991; Feldman and Aoki, 1992), we assume an equilibrium distribution with respect to allele frequencies based on the Hardy-Weinberg principle in the initial generation. The proportion of deaf individuals in the first generation $(d)$ is 0.02 . To achieve an equilibrium distribution, the proportion of hearing carriers of the deaf allele in the initial generation $(c)$ is 0.24 . The model is run for 50 generations $(g)$.

In experiment 1 , assortative marriage $(m)$ is varied and sign language transmission $\left(l_{\mathrm{a}}\right)$ consists of vertical transmission alone. In experiment 2 , assortative marriage $(m)$ is not present, and instead, marriages between deaf individuals are prohibited. Experiment 2 focuses on the different modes of sign language transmission (vertical, horizontal, oblique and grandparental transmission) and the role of hearing signers on sign language persistence.

Experiment 1: assortative marriage. In experiment 1, we investigate the role of assortative marriage on sign language persistence with vertical transmission alone. Aoki and Feldman (1991) find that assortative mating is important for sign language persistence. The assortative marriage parameter used here specifies the proportion of marriages that occur between deaf agents and between hearing agents. For example, if the assortative marriage parameter is set to 0.4 , then $40 \%$ of deaf agents marry a deaf agent and $40 \%$ of hearing agents marry a hearing agent. The remaining $60 \%$ of agents are married randomly, with no preference regarding the deaf/hearing status of their partners.

Here, we conduct an exploration of the effect of this parameter on sign language persistence with the following values for $m: 0.0$, $0.1,0.2,0.3,0.4,0.5,0.6,0.7,0.8,0.9,1.0$. When the value for assortative marriage is 0.0 , all agents are randomly married with respect to the deaf/hearing status of others. Thus, there is a chance that a deaf agent marries another deaf agent. This chance is slim, as the proportion of deaf agents in the population is small given the current parameter settings. There are documented sign languages where marriages between deaf individuals do not occur (e.g. ABSL and AdaSL). To ensure that no marriages occur between deaf individuals, we implement a marriage parameter which ensures that all deaf agents marry a hearing agent (no_dd).

In this set of experiments, the parameter settings are the following: vertical transmission of the sign language occurs from deaf signing parents to deaf children, the proportion of hearing agents acquiring the sign language $(h)$ is 0 , the total population size is 3000 (one generation, $n$, is 1000 ), the proportion of deaf individuals in generation $1(d)$ is 0.2 , the proportion of hearing

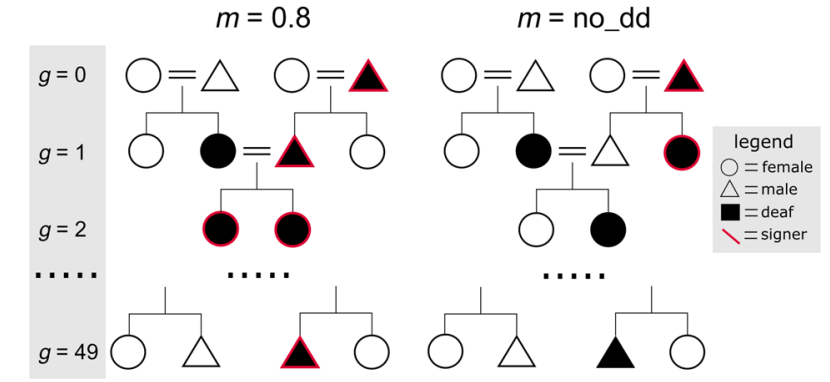

Fig. 2 Two hypothetical model runs showing a few agents per generation in experiment 1 (vertical transmission only and no hearing signers) with assortative marriage $(m)$ set to $0.8(m=0.8)$ and when deaf-deaf marriages are prohibited ( $\boldsymbol{m}=$ no_dd). Deaf agents are in black and hearing agents are in white. The genetic makeup of these agents (homozygous recessive, heterozygous, homozygous dominant) is not shown here. Agents who have acquired the sign language are outlined in red. The graphic illustrates why it is more likely that sign language persists when the value of assortative marriage is higher (here, $m=0.8$ ) compared to when deaf-deaf marriages do not occur $(m=$ no_dd).

carriers of the deaf allele $(c)$ is 0.24 and the number of generations in the simulation $(g)$ is 50 . The parameter under investigation, assortative marriage $(m)$, is varied in a set of experiments. Two hypothetical runs of the model, one with $m=0.8$ and one with $m=$ no_dd, are presented in Fig. 2.

We find that the sign language persists in these experiments when the assortative marriage value is set between 0.6 and 0.9 , shown in Fig. 3. To understand this result, it is necessary to consider the connection between genes causing deafness, the cultural transmission of the sign language and marriage norms. As discussed in Mudd et al. (in press), deaf-deaf marriages always yield deaf offspring. In this model, we only consider hereditary deafness (as opposed to deafness due to other causes), a simplification made for modeling purposes. Though non-congenital deafness may also be present in shared signing communities, it is the unusually high incidence of hereditary deafness which characterizes these communities (Nyst, 2012). Because deaf-deaf marriages always yield deaf offspring in the model, high values of assortative marriage ensure sign language persistence. On the contrary, when the assortative marriage parameter is low (under 0.6), less marriages between deaf agents occur (as compared to those when $m$ is above 0.5), thus less deaf agents are less likely to be born, and thus the sign language is less likely to be transmitted.

This result will be explained using two hypothetical model runs illustrated in Fig. 2, with $m=0.8$ (left) and $m=$ no_dd (right). The first generation $(g=0)$ of each hypothetical run starts with the same configuration: two couples, each with one deaf, signing male (the deaf agents in the first generation are always signers). In the second generation $(g=1)$, when $m=0.8$, the deaf agents marry and have two deaf offspring. The signing father transmits the sign language to the two daughters. When $m=$ no_dd, a deaf agent marries a hearing agent. They have a deaf daughter and a hearing daughter (due to randomly choosing a gene from each parent to determine the offspring's genetic composition). Because neither parent is a signer, the deaf daughter does not acquire the sign language. In the last generation $(g=49)$, deaf agents are more likely to be signers when $m=0.8$, compared to when $m=$ no_dd.

This example highlights the effect of marriage patterns on sign language persistence. We see a clear interaction between genes and culture: marriage norms shape partner choice, which in turn affects the likelihood of having a deaf offspring, which in turn affects sign language transmission (because here, sign language transmission only occurs from deaf parents to deaf offspring). 

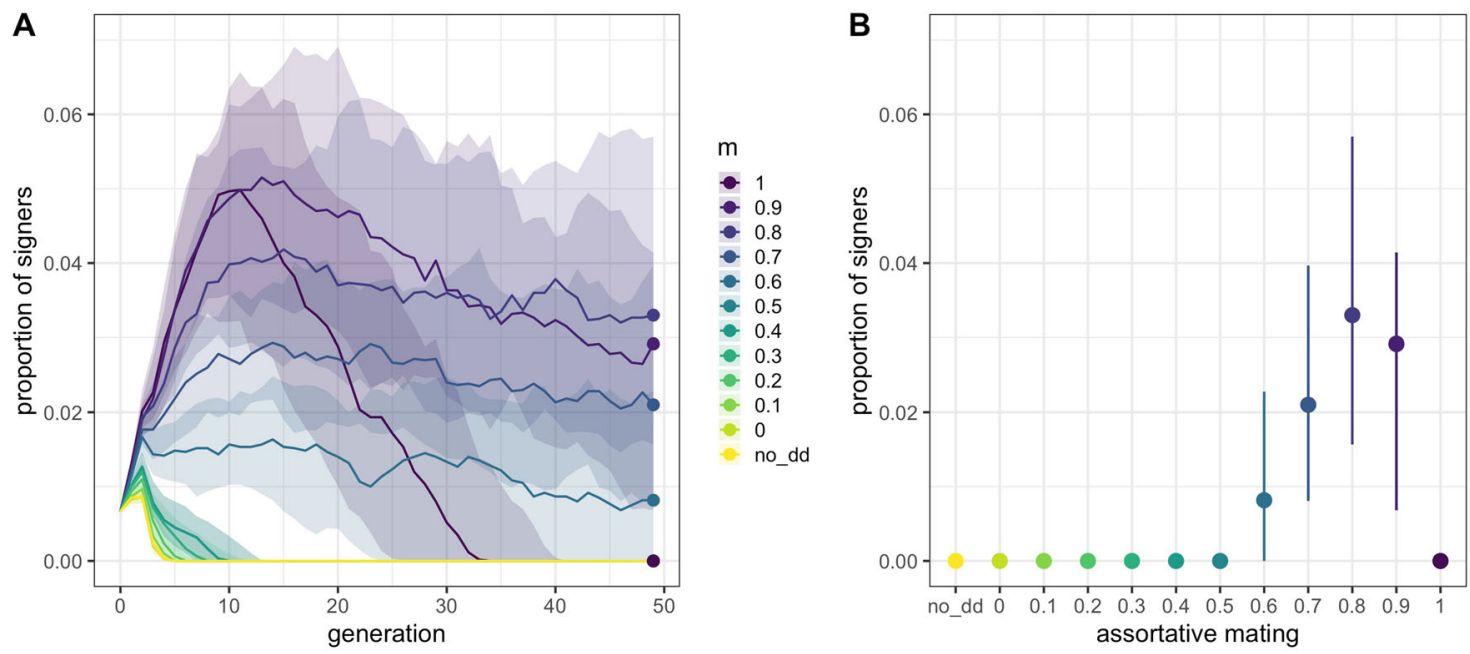

Fig. 3 Experiment 1 considers the effect of the assortative marriage parameter $(m)$ on sign language persistence, with vertical transmission alone and with no hearing agents acquiring the sign language. a Shows the proportion of signers over 50 generations with varying values of assortative marriage $(m)$. The dark line represents the median at each time step and the shaded area represents the first and third quartiles. b Shows the proportion of signers at generation 50 with varying assortative marriage values $(\mathrm{m})$. The dots represent the median at generation 50 and the line represents the first and third quartiles. Note that the colored dots at generation 50 in $\mathbf{a}$ correspond to the colored dots in $\mathbf{b}$

This example also shows how it would be easier for the sign language to persist with more transmission types and hearing signers. Given different parameter settings with regards to these two factors, when $m=$ no_dd, the deaf female in the third generation $(g=2)$ would have been able to acquire the sign language via grandparental transmission (from the deaf grandfather to his deaf granddaughter) or from a hearing signer (e.g. if her hearing father had acquired the sign language from his deaf, signing father).

However, this does not explain why the sign language does not persist when $m$ is set to 1 . In this case, all deaf agents are married to another deaf agent, and all hearing agents are married to another hearing agent. As discussed in more detail in Mudd et al. (in press), when assortative marriage is complete, the sign language does not persist. This counterintuitive result can be explained by certain constraints imposed in the agent-based model, namely that only female-male marriages occur and siblings cannot marry. Because the number of deaf agents in the population is small, as specified by the parameter settings used in these simulations (in the initial generation, $2 \%$ of the population is deaf), it sometimes happens that a deaf agent does not marry because of the marriage constraints. Hence, these constraints impose a fitness disadvantage for deaf agents, which occur only when the proportion of deaf agents in the population is small. Though these marriage constraints were not present in the mathematical model, we find that they present a slightly more realistic representation of shared signing communities, demonstrating under what circumstances hereditary deafness can be transmitted.

Experiment 2: structured sign language transmission methods. In line with Gialluisi et al. (2013), we continue to investigate the observed case where no deaf-deaf marriages occur ( $m=$ no_dd). This marriage pattern has been reported for the AdaSL community and the ABSL community. As shown by Gialluisi et al. (2013) and in experiment 1, current models of sign language persistence with vertical transmission alone have not been able to explain how these sign languages persist. However, when the values from ABSL were applied to Aoki and Feldman's (1991) model, they were applied without considering methods of sign language transmission other than vertical and also without considering hearing individuals who may take part in a signing community. In experiment 2, we expand on previous research in that we consider both of these factors.

In addition, previous models of sign language transmission modeled random horizontal and oblique transmission (Aoki and Feldman, 1991). Here, we consider structured transmission within the family. Further, we consider all grandparental interactions as opposed to just the maternal grandmother, as modeled by Feldman and Aoki (1992). In experiment 2, we investigate the role of different modes of cultural transmission on sign language persistence. We investigate various possible combinations of vertical, horizontal, oblique and grandparental transmission. In this experiment, vertical transmission is always present as it needs to be there to ensure transmission from generation to generation. For example, horizontal transmission alone would not allow the sign language to persist over multiple generations. We investigate eight different cases: vertical transmission alone (V), vertical and horizontal transmission ( $\mathrm{VH})$, vertical and oblique transmission (VO), vertical and grandparental transmission (VG), vertical, horizontal and oblique transmission (VHO), vertical, horizontal and grandparental transmission (VHG), vertical, oblique and grandparental transmission (VOG), and vertical, horizontal, oblique and grandparental (VHOG). In addition, we consider the role of hearing signers on sign language persistence with the parameter $h$. If a hearing individual can acquire the sign language via any of the modes of cultural transmission specified, they acquire it with probability $h$. We consider the following values for $h: 0.0,0.1,0.2,0.3,0.4,0.5,0.6,0.7,0.8,0.9,1.0$. The value specified for $h$ is applied to all modes of cultural transmission in a given simulation. For instance, in the simulation VG with $h$ set to 0.5 , hearing agents who can acquire the sign language via vertical transmission will acquire the sign language with 0.5 probability and hearing agents who can acquire the sign language via grandparental transmission will acquire the sign language with 0.5 probability. We would like to emphasize that none of these transmission types are realistic in isolation, but to understand the contribution of each, we isolate each (though, keeping vertical transmission) (Fig. 4).

We find that the more modes of cultural transmission present, the lower the proportion of hearing signers is needed for the sign language to persist. With vertical transmission alone $(\mathrm{V})$, the probability of hearing signers acquiring the sign language must be 
V

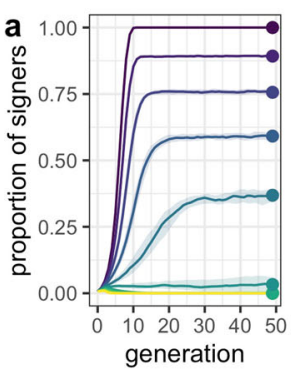

VO

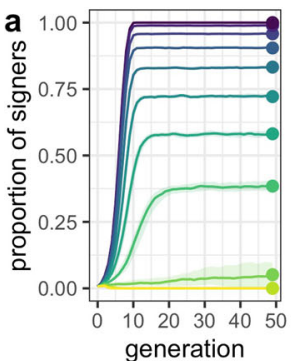

VHO

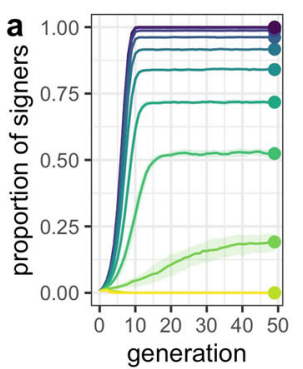

VOG

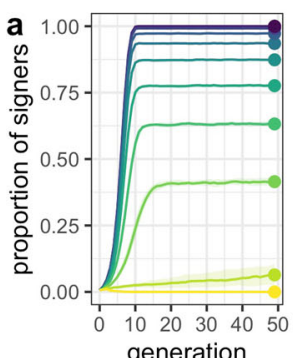

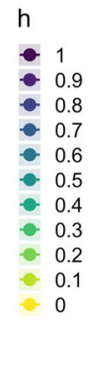
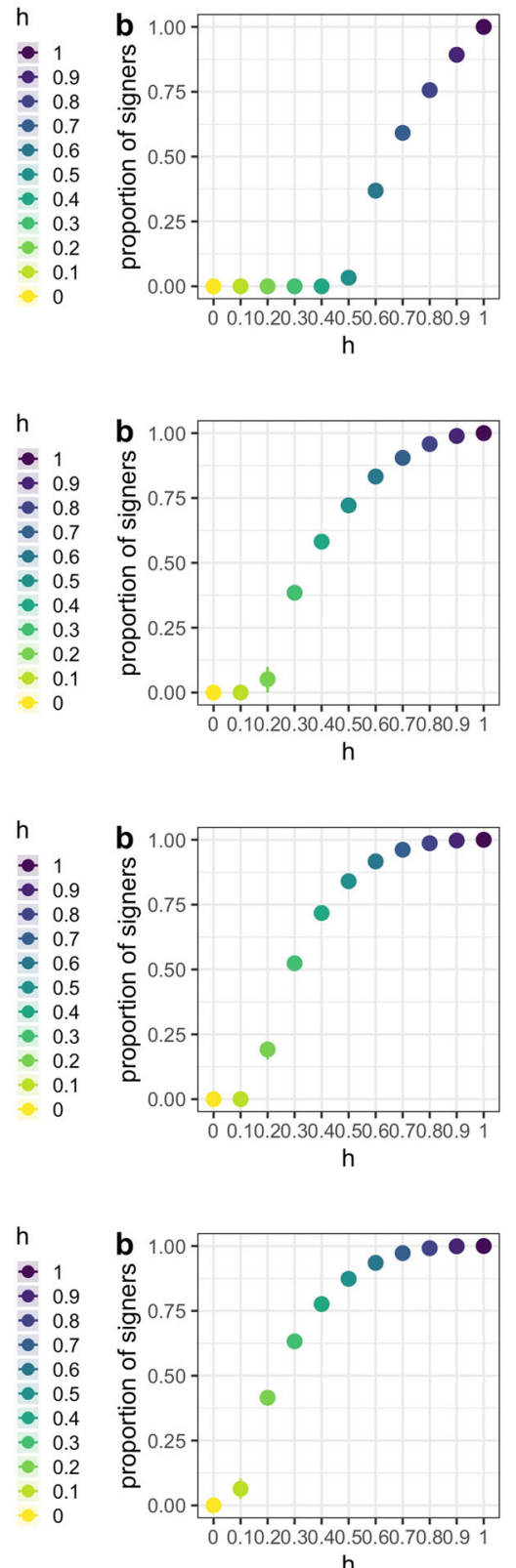

VH

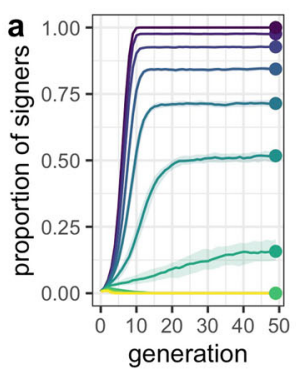

VG

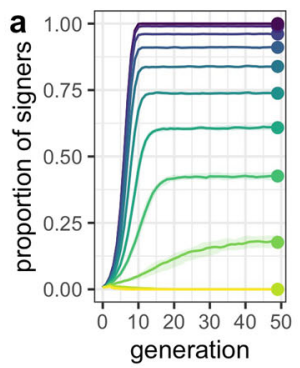

VHG

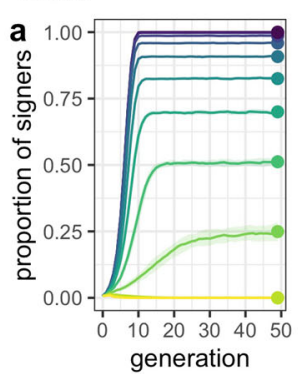

\section{VHOG}
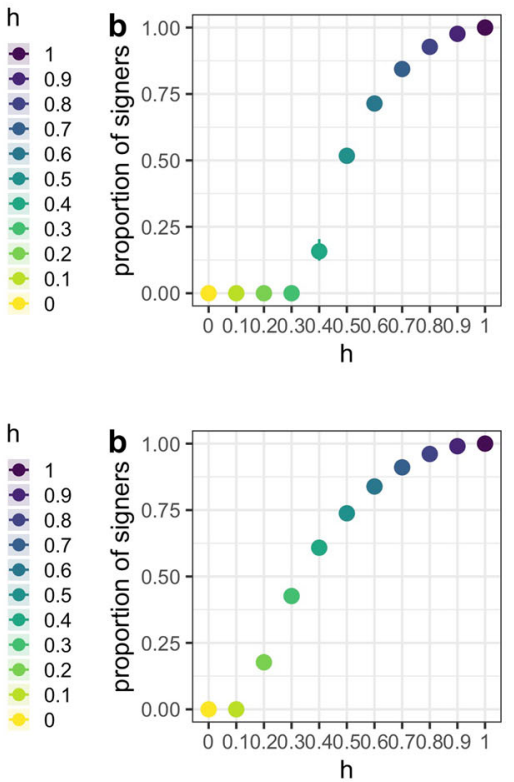

$h$
0.9
0.8
0.7
0.6
0.5
0.4
0.3
0.2
0.1
0
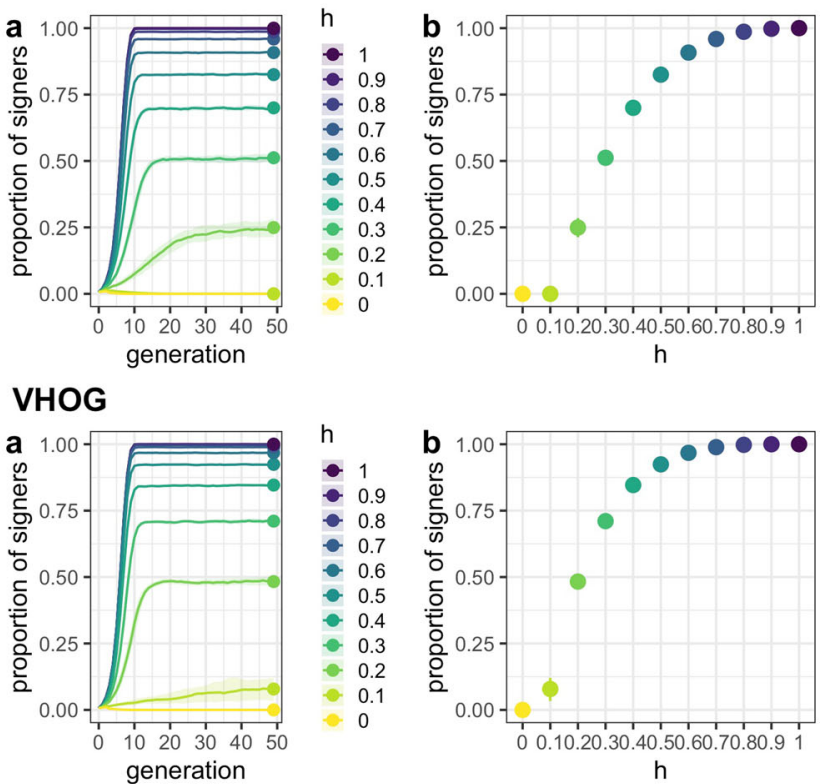
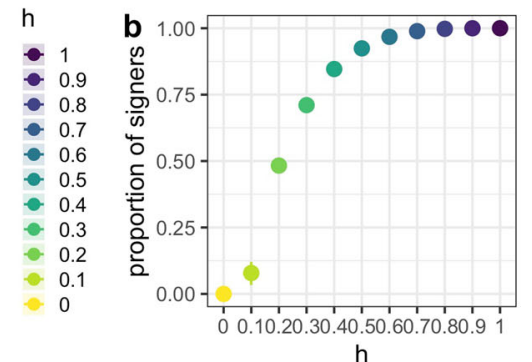

Fig. 4 Experiment 2 considers the effect of different methods of cultural transmission of the sign language (V, VH, VO, VG, VHO, VHG, VOG, and VHOG) and the probability that a hearing agent acquires the sign language $(\boldsymbol{h})$, when no deaf-deaf marriages occur $(\boldsymbol{m}=$ no_dd). For each of these transmission methods, a shows the proportion of signers over 50 generations with varying values of hearing agents acquiring the sign language when possible ( $h$ ). The dark line represents the median at each time step and the shaded area represents the first and third quartiles. $\mathbf{b}$ shows the proportion of signers at generation 50 with varying values of hearing agents acquiring the sign language when possible $(h)$. The dots represent the median at generation 50 and the line represents the first and third quartiles. Note that the colored dots at generation 50 in $\mathbf{a}$ correspond to the colored dots in $\mathbf{b}$.

over 0.5 . We can compare this result to vertical and horizontal transmission $(\mathrm{VH})$, vertical and oblique transmission (VO) and vertical and grandparental transmission (VG). In comparing these separate transmission methods, we see that grandparental transmission and oblique transmission drastically decrease the proportion of hearing signers needed to acquire the sign language for sign language persistence ( $h$ must be over 0.1 ). On the other hand, the effect of adding horizontal transmission is not as extreme: the probability that hearing signers acquire the sign language must be over 0.3 .

Why is horizontal transmission less successful than oblique and grandparental transmission? This is likely because agents can acquire the sign language from more agents via oblique and grandparental transmission than horizontal transmission. With grandparental transmission, agents can acquire from four grandparents: a set of grandparents on the mother's side and a set of grandparents on the father's side. With oblique transmission, the number of agents that an agent may acquire the sign language from depends on the number of siblings their parents have. However, because the siblings of both parents are considered, this number will on average be higher than the number of siblings an agent has. Thus, the finding that grandparental and oblique transmission are more beneficial than horizontal transmission can simply be explained by the number of communication partners an agent can have given the transmission mode. 
In addition, we find that without any hearing individuals partaking in the signing community, the sign language is not predicted to persist. Even when all modes of transmission are present (VHOG), some hearing individuals (over 0\%) need to partake in the signing community for the sign language to persist. In the context of shared sign languages, hearing signers often make up a large percentage of the signing community (Nyst, 2012). Hence, the persistence of shared sign languages where deaf-deaf marriages do not occur can be explained by considering several modes of sign language transmission (more than just vertical transmission) and hearing signers. In shared signing communities, there is usually a high proportion of hearing signers and sign language transmission occurs in the presence of adults and peers (Nyst, 2012). For instance, over half of the hearing population in a rural village in the north of Bali, Indonesia, knows the local sign language, Kata Kolok (Marsaja, 2008). Further, in a shared signing community in Thailand, Ban Khor sign language is used by over $10 \%$ of hearing individuals (Nonaka, 2007). Though there exists variation in the proportion of hearing individuals partaking in the signing community, a number of hearing signers has always been reported in shared signing communities. To conclude, considering various modes of cultural transmission and hearing signers in models of sign language persistence makes for a more realistic representation of how shared sign languages persist. Future work could consider the effect of the high proportion of hearing signers on the features of the sign language and the many other factors which need to be considered in models of shared sign language persistence (Gialluisi et al., 2013; for the case of Kata Kolok see Lutzenberger, submitted).

\section{General discussion}

The discussed mathematical models by Aoki and Feldman (1991) and Feldman and Aoki (1992) consisted of the majority of factors that were considered in the current investigation of sign language persistence. However, the aforementioned models did not consider all of the relevant modes of cultural transmission and hearing signers at once. For example, Feldman and Aoki (1992) just considered vertical and grandparental transmission without hearing signers. Here, we use an agent-based model in which we implement various modes of cultural transmission, hearing signers and marriage patterns. In experiment 1 , we show that sign language persistence is affected by marriage patterns, which we model by an assortative marriage parameter determining the tendency for deaf agents to marry a deaf agent and hearing agents to marry a hearing agent. With vertical transmission alone from deaf parents to deaf children, we find that the sign language only persists when the assortative marriage parameter is between 0.6 and 0.9. This experiment affirms the findings of Aoki and Feldman's (1991) model (1) and also the case of ABSL applied to the model by Gialluisi et al. (2013). In experiment 2, deaf-deaf marriages are prohibited, to model cases like ABSL and AdaSL. Previous models have shown that a high degree of assortative mating is required for sign language persistence (e.g. Aoki and Feldman, 1991), so what is necessary for sign languages to persist when deaf-deaf marriages do not occur? The result of experiment 2 is twofold: we highlight the importance of including more modes of cultural transmission and hearing signers in models of sign language persistence. We show that by adding more modes of cultural transmission of the sign language to the model, the amount of hearing signers necessary for sign language persistence is lessened (but a small proportion of hearing signers is still required).

This model is of course far from realistic in representing the full complexity of shared signing communities. Realism is not the point of the present model, nor for most models (Smaldino, 2017; Epstein, 2008). For a discussion of what could be (still) lacking in explaining sign language persistence in shared signing communities, see Gialluisi et al. (2013). What is particularly unrealistic about the agent-based model (and the previously discussed mathematical models) relates to properties of the teacher and learner. In these models, the deaf/hearing status of only the language learner is considered. Hearing agents acquire the sign language with a certain probability, and (in the agent-based model presented) deaf agents always acquire the sign language if they can. However, the deaf/hearing status of the teacher is ignored. Consider an example of vertical transmission with a deaf parent and a hearing child. In these models, only the deaf/hearing status of the offspring is considered, so the hearing child will learn the sign language with a given probability regardless of having a deaf teacher (in this case, their parent). What is much more realistic, is that the deaf status of the teacher and learner are both taken into account. In real life, a hearing offspring is likely to acquire the sign language if they have a deaf parent (Bishop and Hicks, 2009). In a subsequent model (Mudd et al., in press) we attempt to make the cultural transmission process more accurate by considering the entire family as a linguistic unit which either acquires or does not acquire the sign language, depending on whether there is a deaf family member and a family member who knows the sign language. In addition, Mudd et al. (in press) consider how sign language persistence is affected by other factors, such as population size and the proportion of hearing agents who are carriers of the gene causing deafness.

The goal of the present research was to understand the role that the various modes of cultural transmission play on sign language persistence. Like Aoki and Feldman (1991) we found that vertical transmission is important, as well as grandparental transmission (also found by Feldman and Aoki, 1992). Additionally, (unlike Aoki and Feldman, 1991) we find that horizontal and oblique transmission, when structured in the family, do help sign language persistence. Shared sign languages present a unique example of ongoing gene-culture coevolution: sign language transmission and the genetic transmission of alleles for deafness coevolve in marriage patterns. Laland et al. (2010), models of gene-culture coevolution provide an interdisciplinary approach to the study of human evolution, by allowing for the integration of genetic and cultural data. We have used this approach to model the coevolution of sign language and deafness.

\section{Conclusion}

To conclude, previous models of sign language persistence have neglected key features of shared sign languages, including various modes of sign language transmission and the often high proportion of hearing signers. Here, we implement Aoki and Feldman's (1991) mathematical model of sign language persistence as an agent-based model to test the role of assortative marriage, sign language transmission methods and hearing signers. Specifically, we focus this study on different modes of sign language transmission-vertical, horizontal, oblique and grandparental-which are implemented in a structured way within the family. We find that, in the absence of deaf-deaf marriages (a marriage pattern reported for several shared signing communities), the threshold for sign language persistence is lowered by a maximum number of sign language transmission methods and a high proportion of hearing signers. These results highlight the importance of having as many methods of cultural transmission as possible for the persistence of a cultural entity (here, sign language). Additionally, the persistence of shared sign languages provides an exceptionally well-documented modern case of gene-culture coevolution. 


\section{Data availability}

The model code is available as supplementary material at: https:// doi.org/10.6084/m9.figshare.11522589.v5.

Received: 6 January 2020; Accepted: 23 April 2020;

Published online: 01 June 2020

\section{References}

Acerbi A, Parisi D (2006) Cultural transmission between and within generations. J Artif Soc Soc Simul 9(1):9

Aoki K, Feldman MW (1991) Recessive hereditary deafness, assortative mating, and persistence of a sign language. Theor Popul Biol 39(3):358-372

Aoki K, Feldman MW (1994) Cultural transmission of a sign language when deafness is caused by recessive alleles at two independent loci. Theor Popul Biol 45(1):101-120

Arnos KS, Welch KO, Tekin M, Norris VW, Blanton SH, Pandya A, Nance WE (2008) A comparative analysis of the genetic epidemiology of deafness in the United States in two sets of pedigrees collected more than a century apart. Am J Hum Genet 83(2):200-207

Bersaglieri T, Sabeti PC, Patterson N, Vanderploeg T, Schaffner SF, Drake JA, Rhodes M, Reich DE, Hirschhorn JN (2004) Genetic signatures of strong recent positive selection at the lactase gene. Am J Hum Genet 74 (6):1111-1120

Bishop M, Hicks SL (2009) Hearing, mother father deaf: hearing people in deaf families. Gallaudet University Press

Cavalli-Sforza LL, Feldman MW (1981) Cultural transmission and evolution: a quantitative approach. Princeton University Press

Coop G, Bullaughey K, Luca F, Przeworski M (2008) The timing of selection at the human FOXP2 gene. Mol Biol Evol 25(7):1257-1259

Epstein JM (2008) Why model? J Artif Soc Soc Simul 11(4):12

Feldman MW, Cavalli-Sforza LL (1976) Cultural and biological evolutionary processes, selection for a trait under complex transmission. Theor Popul Biol $9(2): 238-259$

Feldman MW, Aoki K (1992) Assortative mating and grandparental transmission facilitate the persistence of a sign language. Theor Popul Biol 42 (2):107-116

Feldman MW, Laland KN (1996) Gene-culture coevolutionary theory. Trends Ecol Evol 11(11):453-457

Fraser GR (1976) The causes of profound deafness in childhood. Johns Hopkins University Press

Gagne DL (2017) With a little help from my friends: the contributions of a peer language network on the conventionalization of space in an emerging language. Doctoral Dissertations, University of Connecticut

Gialluisi A, Dediu D, Francks C, Fisher SE (2013) Persistence and transmission of recessive deafness and sign language: new insights from village sign languages. Eur J Hum Genet 21(9):894-896

Gong T, Shuai L (2016) Simulating the effects of cross-generational cultural transmission on language change. In: Mehler A, Lücking A, Banisch S, Blanchard P, Job B (eds) Towards a theoretical framework for analyzing complex linguistic networks. Springer, Berlin, Heidelberg, pp. 237-256

Gong T, Minett JW, Wang WS-Y (2010) A simulation study exploring the role of cultural transmission in language evolution. Connect Sci 22(1):69-85

Grimm V, Berger U, DeAngelis DL, Polhill JG, Giske J, Railsback SF (2010) The ODD protocol: a review and first update. Ecol Model 221:2760-2768

Grimm V, Berger U, Bastiansen F, Eliassen S, Ginot V, Giske J, DeAngelis DL (2006) A standard protocol for describing individual-based and agent-based models. Ecol Model 198:115-126

Hewlett BS, Cavalli-Sforza LL (1986) Cultural transmission among Aka Pygmies. Am Anthropologist 88(4):922-934

Holden C, Mace R (2009) Phylogenetic analysis of the evolution of lactose digestion in adults. Hum Biol 81(5-6):597-619

Kirby S (2001) Spontaneous evolution of linguistic structure-an iterated learning model of the emergence of regularity and irregularity. IEEE Trans Evolut Comput 5(2):102-110

Kirby S, Hurford JR (2002) The emergence of linguistic structure: an overview of the iterated learning model. In: Cangelosi A, Parisi D (eds) Simulating the evolution of language. Springer, London, pp. 121-147

Kirby S, Cornish H, Smith K (2008) Cumulative cultural evolution in the laboratory: an experimental approach to the origins of structure in human language. PNAS 105(31):10681-10686

Kirby S (2000) Syntax without natural selection: how compositionality emerges from vocabulary in a population of learners. In: Knight C, Studdert-Kennedy M, Hurford J (eds) The Evolutionary Emergence of Language: Social Function and the Origins of Linguistic Form. Cambridge University Press, Cambridge, United Kingdom
Kisch S (2012) Al-Sayyid: a sociolinguistic sketch. In: Zeshan U, de Vos C (eds) Sign languages in village communities: anthropological and linguistic insights. Mouton De Gruyter, Berlin, pp. 365-372

Kusters A (2012) Adamorobe: a demographic, sociolinguistic and sociocultural profile. In: Zeshan U, de Vos C (eds) Sign languages in village communities: anthropological and linguistic insights. Mouton De Gruyter, Berlin, pp. 347-352

Kyle JW, Woll SB (1985) Sign language: the study of deaf people and their language, Cambridge University Press, Cambridge, United Kingdom

Laland KN, Odling-Smee J, Myles S (2010) How culture shaped the human genome: bringing genetics and the human sciences together. Nat Rev Genet 11 (2):137-148

Laland KN, Kumm J, Van Horn JD, Feldman MW (1995) A gene-culture model of human handedness. Behav Genet 25(5):433-445

Lutzenberger $\mathrm{H}$ (submitted) Threat or natural fluctuation? Revisiting language vitality of Kata Kolok, the sign language of a village in Bali. UNESCO at the occasion of 2019 International year of indigenous languages

Marsaja IG (2008) Desa Kolok: A deaf village and its sign language in Bali, Indonesia, Ishara Press, Nijmegen, the Netherlands

Meir I, Sandler W, Padden C, Aronoff M (2010) Emerging sign languages. In: Marschark M, Spencer PA (eds) The Oxford handbook of deaf studies, language, and education. Oxford University Press, New York, pp. 267-280

Meir I, Israel A, Sandler W, Padden CA, Aronoff M (2012) The influence of community on language structure: evidence from two young sign languages. Linguist Var 12(2):247-291

Motamedi Y, Schouwstra M, Smith K, Culbertson J, Kirby S (2019) Evolving artificial sign languages in the lab: from improvised gesture to systematic sign. Cognition 192:103964

Mudd K, de Vos C, de Boer B (in press) An agent-based model of sign language persistence informed by real-world data. Language Dynamics and Change

Nance WE, Kearsey MJ (2004) Relevance of connexin deafness (DFNB1) to human evolution. Am J Hum Genet 74(6):1081-1087

Nance WE, Liu X-Z, Pandya A (2000) Relation between choice of partner and high frequency of connexin-26 deafness. Lancet 356(9228):500-501

Nonaka A (2007) Emergence of an indigenous sign language and a speech/sign community in Ban Khor, Thailand. PhD dissertation, University of California, Los Angeles.

Nyst V (2012) Shared sign languages. In: Pfau R, Steinbach M, Woll B (eds) Sign language: an international handbook. Mouton de Gruyter, Berlin, pp. 552-574

O'Rourke DH (2018). Hardy-Weinberg equilibrium. The International Encyclopedia of Biological Anthropology, American Cancer Society, pp. 1-2

Petit C (1996) Genes responsible for human hereditary deafness: symphony of a thousand. Nat Genet 14(4):385-391

Senghas A (2005) Language emergence: clues from a new Bedouin sign language. Curr Biol 15(3):463-465

Senghas A, Kita S, Özyürek A (2004) Children creating core properties of language: evidence from an emerging sign language in Nicaragua. Science 305 (5691):1779-1782

Smaldino PE (2017) Models are stupid, and we need more of them. In: Vallacher RR, Read SJ, Nowak A (eds) Computational social psychology. Routledge, New York, pp. 311-331

Smith K, Hurford JR (2003) Language evolution in populations: extending the iterated learning model. In: Banzhaf W, Ziegler J, Christaller T, Dittrich P, Kim JT (eds) Advances in artificial life. Springer, Berlin, pp. 507-516

Theisen-White C, Kirby S, Oberlander J (2011) Integrating the horizontal and vertical cultural transmission of novel communication systems. In: Carlson L, Hölscher C, Shipley T (eds) Proceedings of the 33rd Annual Conference of the Cognitive Science Society. Cognitive Science Society, Boston, pp. 956-961

Williamson SH, Hubisz MJ, Clark AG, Payseur BA, Bustamante CD, Nielsen R (2007) Localizing recent adaptive evolution in the human genome. PLoS Genet 3(6):0902-0915

Winata S, Arhya IN, Moeljopawiro S, Hinnant JT, Liang Y, Friedman TB, Asher JH (1995) Congenital non-syndromal autosomal recessive deafness in Bengkala, an isolated Balinese village. J Med Genet 32(5):336-343

Woll B, Sutton-Spence R, Elton F (2001) Multilingualism: the global approach to sign language. In: Lucas C (ed) The sociolinguistics of sign languages Cambridge University Press, Cambridge

Zeshan U, de Vos C (2012) Sign languages in village communities, anthropological and linguistic insights. De Gruyter Mouton, Berlin, Boston

\section{Acknowledgements}

A special thanks to Bill Thompson, Sean Roberts, and Yannick Jadoul for providing information from a related project modeling Kata Kolok sign fluency across the community (de Vos et al., 2016). Thanks to Yannick Jadoul and Marnix van Soom for coding help and fruitful feedback throughout the project. This research was supported by the FWO-NWO grant "The emergence of phonology within six generations" awarded to Bart 
de Boer, Paula Fikkert, and Connie de Vos, the Flemish AI plan, and the NWO Veni grant "The face in sign language interaction" awarded to Dr. de Vos.

\section{Competing interests}

The authors declare no competing interests.

\section{Additional information}

Correspondence and requests for materials should be addressed to K.M.

Reprints and permission information is available at http://www.nature.com/reprints

Publisher's note Springer Nature remains neutral with regard to jurisdictional claims in published maps and institutional affiliations. (c) (i) Open Access This article is licensed under a Creative Commons Attribution 4.0 International License, which permits use, sharing, adaptation, distribution and reproduction in any medium or format, as long as you give appropriate credit to the original author(s) and the source, provide a link to the Creative Commons license, and indicate if changes were made. The images or other third party material in this article are included in the article's Creative Commons license, unless indicated otherwise in a credit line to the material. If material is not included in the article's Creative Commons license and your intended use is not permitted by statutory regulation or exceeds the permitted use, you will need to obtain permission directly from the copyright holder. To view a copy of this license, visit http://creativecommons.org/ licenses/by/4.0/.

(C) The Author(s) 2020 